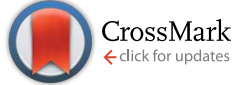

Cite this: RSC Adv., 2015, 5, 100689

Received 23rd September 2015 Accepted 17th November 2015

DOI: 10.1039/c5ra19654j

www.rsc.org/advances

\title{
Potential responses to neutral thiophenols of polymeric membrane electrodes and their applications in potentiometric biosensing $\dagger$
}

\author{
Long Li $i^{\mathrm{ab}}$ and Wei Qin*a
}

The unexpected potential responses to electrically neutral thiophenols (ArSHs) of anion-exchanger doped polymeric membranes are described. Based on the dimerization reactions of ArSHs via horseradish peroxidase (HRP)-catalyzed oxidations, a sensitive and facile potentiometric biosensing platform for HRP has been developed.

For many decades, polymeric membrane ion-selective electrodes have been considered to be feasible only for ionic analytes because a charged species can form a charge separation layer at the interface between a membrane phase and an aqueous phase. ${ }^{1}$ This has significantly limited the development and applications of potentiometric membrane electrodes for detection of electrically neutral species and sensing of their related reactions. In recent years, the potential response behaviors and mechanisms of several kinds of electrically neutral species such as phenols (ArOHs) ${ }^{2}$ and boronic acids $(\mathrm{BAs})^{3}$ on the ion exchanger-doped polymeric membranes have been reported. It has been found that phenols and boronic acids can form the complexes with quaternary ammonium salts via hydrogen bonding in the membrane phase, which could undergo proton dissociation with the concomitant ejection of the formed acids from the membrane phase into the aqueous phase. ${ }^{2,3}$ Based on the oxidations of monomeric phenols to oligomeric phenols and reversible covalent bindings between boronic acid groups and diols, potentiometric sensing platforms for horseradish peroxidase (HRP) mimetics ${ }^{4}$ and for saccharides $^{3,5}$ have been developed, respectively. However, electrically neutral species that can induce potential responses on the polymeric membrane electrodes are rather limited. ${ }^{2,3}$

${ }^{a}$ Key Laboratory of Coastal Environmental Processes and Ecological Remediation, Yantai Institute of Coastal Zone Research (YIC), Chinese Academy of Sciences (CAS), Shandong Provincial Key Laboratory of Coastal Environmental Processes, YICCAS, Yantai, Shandong 264003, P. R. China. E-mail: wqin@yic.ac.cn; Fax: +86 535 2109000; Tel: +865352109156

${ }^{b}$ University of the Chinese Academy of Sciences, Beijing 100049, P. R. China

$\dagger$ Electronic supplementary information (ESI) available. See DOI: $10.1039 / \mathrm{c} 5 \mathrm{ra} 19654 \mathrm{j}$
More examples are still highly desired because they are favourable to ascertain the generality of this phenomenon and the corresponding response mechanism. Moreover, more electrically neutral species that can induce potential responses on the polymeric membrane electrodes will largely broaden the applications of potentiometric sensors and stimulate the research on electrochemistry at the interface between two immiscible phases.

Thiols are an important class of molecules in chemical industry and biological systems. ${ }^{6}$ Thiophenols (ArSHs) have been widely used in the production of agrochemicals, polymers and pharmaceuticals. ${ }^{7}$ Aliphatic thiols such as cysteine, homocysteine and glutathione involved in many physiological processes play important roles in living organisms. ${ }^{7}$ ArSHs are more toxic than aliphatic thiols, and it is essential to selectively differentiate ArSHs from aliphatic thiols. ${ }^{6,8}$ Based on the stronger nucleophilicities of the corresponding thiolates from ArSHs, selective fluorescent probes for ArSHs can be developed. ${ }^{8,9}$ However, those fluorescent probes are usually structurally complicated, and the synthesis procedures are time consuming.

In this work, the unexpected potential responses to electrically neutral ArSHs of polymeric membranes doped with lipophilic anion exchangers are shown. The complexations between ArSHs and quaternary ammonium salts have been studied in homogeneous organic solutions. Efforts have been made to relate the response behaviors of ArSHs to their lipophicities and the association constants between ArSHs and tetraalkylammonium chloride. The heterogeneous two phase extraction experiments indicate the dissociation behaviors of ArSHs in the organic phase and confirm the response mechanism. The potential responses to ArSHs of the polymeric membranes provide a simple way to differentiate ArSHs from biologically important bioactive aliphatic thiols. By using ArSHs as reducing substrates, a potentiometric sensing platform for oxidases and their related reactions can be developed.

Polymeric liquid membranes containing poly(vinyl chloride) (PVC) and $o$-nitrophenyl octylether (o-NPOE) in a weight ratio of 


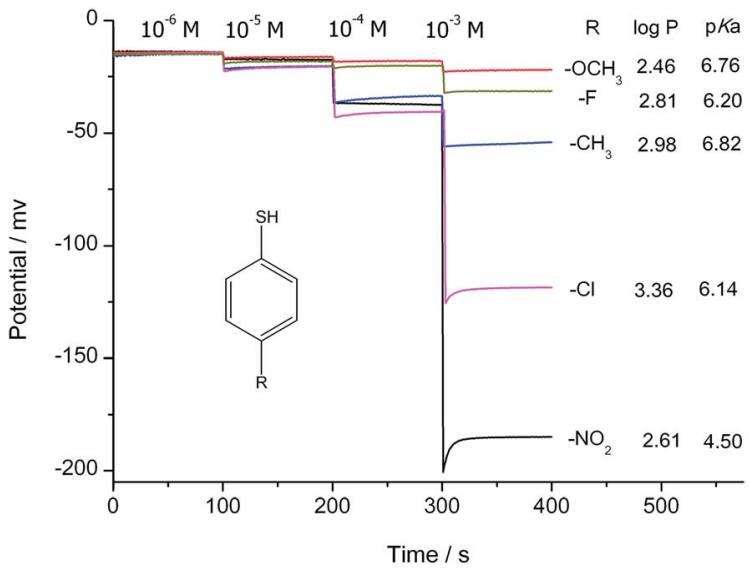

Fig. 1 Potential responses to five ArSHs in a $50 \mathrm{mM}$ acetate buffer of $\mathrm{pH} 4.0$ at the o-NPOE-PVC-TDMA ${ }^{+} \mathrm{Cl}^{-}$membrane electrode. All the $\mathrm{p} K_{\mathrm{a}}$ and $\log P$ values were calculated by $\mathrm{ACD} / \mathrm{Labs} 12$. All potential values were measured using an electrochemical workstation under zero current conditions.

$1: 1$ and the receptor salt $\left(10 \mathrm{mM} \mathrm{kg}{ }^{-1}\right)$ were prepared by the solvent-casting technique with tetrahydrofuran as the casting solvent. After transferring the cocktail into a glass ring fixed on a glass plate and letting tetrahydrofuran evaporate overnight, a uniform membrane of $200 \mu \mathrm{m}$ thickness was obtained. Disks of $5 \mathrm{~mm}$ diameter were punched from the parent membrane and glued to plasticized PVC tubes (i.d. $3 \mathrm{~mm}$, o.d. $5 \mathrm{~mm}$ ) to fabricate the polymeric membrane electrodes. Fig. 1 shows the potential responses to a number of ArSHs of a tridodecylmethylammonium chloride $\left(\mathrm{TDMA}^{+} \mathrm{Cl}^{-}\right)$-doped polymeric membrane. At $\mathrm{pH}$ 4.0, most of the ArSHs exist in their undissociated (unionized) forms. The potential differences in the concentration range of $10^{-4}-10^{-3} \mathrm{M}$ were -90 and $-140 \mathrm{mV}$ for $-\mathrm{Cl}$ and $-\mathrm{NO}_{2}$ substituted ArSHs, respectively, which are larger than the theoretical value for a singly charged anion. The anionic potential responses induced by the electrically neutral ArSHs are unexpected, since neutral species cannot perturb the distribution of charged species directly at the interface between the immiscible phases. ${ }^{10}$ It has been found that phenols and boronic acids can undergo proton dissociation in the organic phase with concomitant ejection of HX to the aqueous phase, thus inducing the anionic responses at the sample-membrane interface. ${ }^{2,3}$

To investigate the response mechanism of the membrane electrode for ArSHs, efforts were made to understand the interactions between ArSHs and quaternary ammonium salts in homogeneous organic solutions. The association constants $\left(K_{\mathrm{a}}\right)$ for the $1: 1$ complexes between ArSHs and TDMA ${ }^{+} \mathrm{Cl}^{-}$were measured in chloroform solutions using UV titration experiments (Fig. S1, ESI $\dagger$ ). As shown in Table 1, the stabilities of the complexes can be determined by the acidities $\left(\mathrm{p} K_{\mathrm{a}}\right)$ of the ArSH molecules, and the calculated data correlate well with the corresponding Hammett constants for the substituents (Fig. S2, ESI $\dagger$ ). ${ }^{12}$ It should also be noted that the $K_{\mathrm{a}}$ values for the complexes between ArSHs and $\mathrm{TDMA}^{+} \mathrm{Cl}^{-}$are significantly larger than those for ArOHs, probably due to the higher
Table 1 Association constants $\left(K_{\mathrm{a}}\right)$ for ArSHs or $\mathrm{ArOH}$, acid dissociation constants $\left(\mathrm{p} K_{\mathrm{a}}\right)$ for ArSHs and Hammett constants $(H)$ for different substituents

\begin{tabular}{llll}
\hline ArSH & $K_{\mathrm{a}}\left(\mathrm{M}^{-1}\right)$ & $\mathrm{p} K_{\mathrm{a}}{ }^{c}$ & $H^{d}$ \\
\hline$p$-Nitro(thio)phenol & ${ }^{a} 4.3 \times 10^{5} / /^{b} 1.3 \times 10^{5}$ & 4.5 & 0.78 \\
$p$-Fluorothiophenol & $2.4 \times 10^{5}$ & 6.2 & 0.06 \\
$p$-Chlorothiophenol & $1.5 \times 10^{5}$ & 6.1 & 0.23 \\
$p$-Toluenethiol & $1.3 \times 10^{5}$ & 6.8 & -0.17 \\
$p$-Methox(thio)phenol & ${ }^{a} 1.7 \times 10^{5} /^{b} 7.9 \times 10^{3}$ & 6.8 & -0.27
\end{tabular}

${ }^{a}$ The association constants of the $1: 1$ complexes for ArSHs with $\mathrm{TDMA}^{+} \mathrm{Cl}^{-}$were determined at room temperature $\left(c a .25^{\circ} \mathrm{C}\right)$ by $\mathrm{UV}$ titration experiments. ${ }^{b}$ Association constants for ArOHs and $\mathrm{TDMA}^{+} \mathrm{Cl}^{-}$from ref. 2. ${ }^{c}$ Acid dissociation constants calculated by ACD/Labs 12. ${ }^{d}$ Hammett constants from ref. 12.

acidities of ArSHs. Studies on the complexations between ArOHs and quaternary ammonium salts $\left(\mathrm{Q}^{+} \mathrm{X}^{-}\right)$show that the $K_{\mathrm{a}}$ values are $\mathrm{X}^{-}$dependent but $\mathrm{Q}^{+}$independent. ${ }^{11}$ It can be assumed that the interaction between an undissociated $-\mathrm{SH}$ group and the anionic component $\mathrm{X}^{-}$of $\mathrm{Q}^{+} \mathrm{X}^{-}$may be the main association form for the complexation between $\mathrm{Q}^{+} \mathrm{X}^{-}$and an ArSHs compound. This is reasonable since the dissociated $\mathrm{ArS}^{-}$ group could hardly complex with the bulky $\mathrm{Q}^{+}$group to form an ion pair, let alone the thermodynamically unfavourable product $\mathrm{H}^{+} \mathrm{X}^{-} .13$

Extraction experiments in two phase systems were carried out with aqueous solutions containing $p$-chlorothiophenol and chloroform solutions with or without $\mathrm{TDMA}^{+} \mathrm{Cl}^{-}$. UV spectra were measured to study the extraction behaviors of ArSHs and their deprotonation processes. As shown in Fig. S3 (ESI $\dagger$ ), when $p$-chlorothiophenol in water (at $\mathrm{pH} 4)$ is extracted into pure $\mathrm{CHCl}_{3}$, the $\mathrm{UV}$ spectrum of the $\mathrm{CHCl}_{3}$ phase shows a peak at 250 $\mathrm{nm}$, which originates from the undissociated neutral $p$-chlorothiophenol. However, when $\mathrm{CHCl}_{3}$ with $\mathrm{TDMA}^{+} \mathrm{Cl}^{-}$is used to extract $p$-chlorothiophenol, the peak at $250 \mathrm{~nm}$ decreases along with an increase at $275 \mathrm{~nm}$, indicating the complexation occurs between $p$-chlorothiophenol and $\mathrm{TDMA}^{+} \mathrm{Cl}^{-}$. Experiments also show that the $\mathrm{pH}$ of the aqueous phase decreases from 4.0 to 3.5 while the concentration of chloride increases after extraction of $p$-chlorothiophenol from the aqueous into the $\mathrm{CHCl}_{3}$ phase containing TDDA $^{+} \mathrm{Cl}^{-}$(see Table S1, ESI $\uparrow$ ). According to the $\mathrm{pH}$ change, it can be estimated that $c a .40 \% p$-nitrothiophenol extracted into the organic phase dissociates. The extraction experiments confirm the dissociation of ArSHs in the organic phase and the ejection of $\mathrm{H}^{+} \mathrm{X}^{-}$into the aqueous phase, which causes the deportation process thermodynamically favourable and induces the anionic potential responses. The above results indicate that the response mechanism for ArSHs is similar to those for ArOHs and BAs (Scheme S1, ESI $\dagger$ ). In these processes, BAs behavior as Lewis acids, while ArSH and ArOHs work as Bronsted acids.

In consistency with the potential responses to ArOHs and BAs of the polymeric membranes doped with lipophilic quaternary ammonium salts, the sensitivity of the ArSH membrane electrode is dependent on the acidity $\left(\mathrm{p} K_{\mathrm{a}}\right)$ and lipophilicity $(\log P)$ of each ArSH. As shown in Fig. 1, the 
magnitudes of the potentiometric responses to the ArSHs are in the order of $p-\mathrm{NO}_{2}>p-\mathrm{Cl}>p-\mathrm{CH}_{3}>p-\mathrm{F}>p-\mathrm{OCH}_{3}$. Indeed, a thiophenol derivative with a stronger acidity and higher $\log P$ induces a larger anionic response. The response order of $p-\mathrm{NO}_{2}>p-\mathrm{Cl}>p-\mathrm{CH}_{3}$ is induced by the acidity effect, and the order of $p-\mathrm{Cl}>p-\mathrm{CH}_{3}>p-\mathrm{F}>p-\mathrm{OCH}_{3}$ is due to the lipophilicity effect. Similar potentiometric selectivities were also reported by Umezawa. ${ }^{2}$ It was found that alkylthiols could not induce potential responses on the polymeric membrane (data not shown), probably due to their weak acidities and low lipophilicities. Therefore, it can be concluded that the aromatic thiol moiety is crucial to the potential responses. Considering that alkylthiols cannot induce potential response on the membrane electrodes, the polymeric membrane electrode may be used as a simple and efficient sensor for distinguishing ArSHs from alkylthiols.

The responses of the membranes doped with different quaternary ammonium cations (i.e., $\mathrm{TDMA}^{+}$and $\mathrm{TBuA}^{+}$) and anions with (i.e., $\mathrm{TCPB}^{-}, \mathrm{SCN}^{-}$and $\mathrm{Cl}^{-}$) were investigated. The results are shown in (Fig. S4, ESI $\dagger$ ). In contrast to negligible responses of the blank and the hydrophilic $\mathrm{TBuA}^{+}$doped membranes, all membranes containing lipophilic $\mathrm{TDMA}^{+}$show anionic responses, which are dependent on the hydrophilicities and $\mathrm{H}$-bond basicities of the anions (i.e., $\mathrm{Cl}^{-}>\mathrm{SCN}^{-}>\mathrm{TCPB}^{-}$). The order of the magnitude of potentiometric response is parallel to that of the charge-dipole and/or hydrogen bonding interactions between $\mathrm{X}^{-}$and $\mathrm{ArOH}$ as expected from the ion radiuses of $\mathrm{X}^{-}$(1.8 and $2.08 \AA$ for $\mathrm{Cl}^{-}$and $\mathrm{SCN}^{-}$, respectively ${ }^{2}$. It is clear that lipophilic cations dominate and the anions regulate the potential responses.

ArSHs can be used as oxidase substrates due to their high reducibilities. The oxidations of ArSHs induce $\mathrm{S}-\mathrm{S}$ coupling and no thiol groups remain in the oxidation products. Therefore, these oxidation processes could "turn off" the anionic potential responses of the reporter thiol groups and the negative signals would return to the positive ones. The potentiometric platform for oxidase/mimic enzymes and their related reactions can be developed as shown in Scheme 1. As a proof of concept experiment, the potential traces to 4 kinds of ArSHs at the polymeric membrane electrode before and after the oxidation reactions are shown in Fig. S5 (ESI $\dagger$ ). It can be seen that anionic potential responses can be obtained after ArSHs are added into the solutions, and the positive responses are indeed observed after $\mathrm{H}_{2} \mathrm{O}_{2}$ and HRP are added to induce the oxidation reactions of the ArSHs. These results confirm that the formed S-S coupling

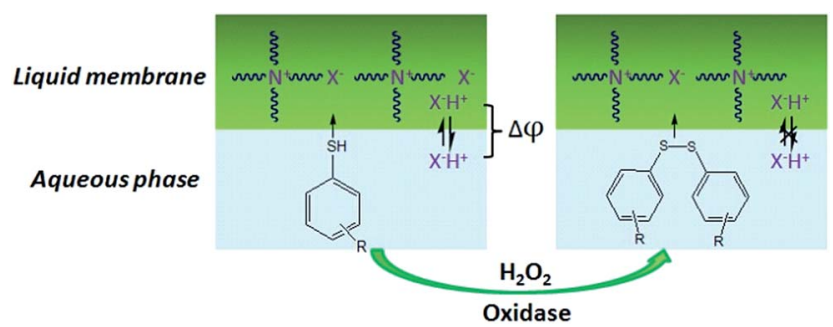

Scheme 1 Response mechanism of the proposed potentiometric platform. products cannot induce potential responses at the polymeric membrane electrodes. Our previous studies indicate that the potentiometric sensing of HRP/mimic enzymes can be done via the oligomerization reactions of ArOHs based on the large lipophicities of the formed oligomeric ArOHs. ${ }^{4}$ Unlike those of ArOHs which involve $\mathrm{C}-\mathrm{O}$ and $\mathrm{C}-\mathrm{C}$ coupling reactions, the oxidations of ArSHs produce the $\mathrm{S}-\mathrm{S}$ coupling products. For the ArOH based platform using the "negative to negative" signal mode, the initial negative potential response could be a large background for the following detection, so that the substrate should be carefully selected to insure not only a rapid reaction kinetics but also a large $\log P$ change. However, for the ArSHs based method using the "negative to positive" signal mode, only a fast reaction kinetics is required since the $\mathrm{S}-\mathrm{S}$ coupling product without the thiol moiety cannot induce any potential response on the polymeric membrane. As shown in Fig. S5, $\dagger$ the large potential change between the responses before and after oxidation reactions indicates that $p$-nitrothiophenol is the most suitable substrate in these 4 kinds of ArSHs for HRP detection.

As a preliminary application, the potentiometric platform was used to detect HRP in the absence and presence of $\mathrm{H}_{2} \mathrm{O}_{2}$
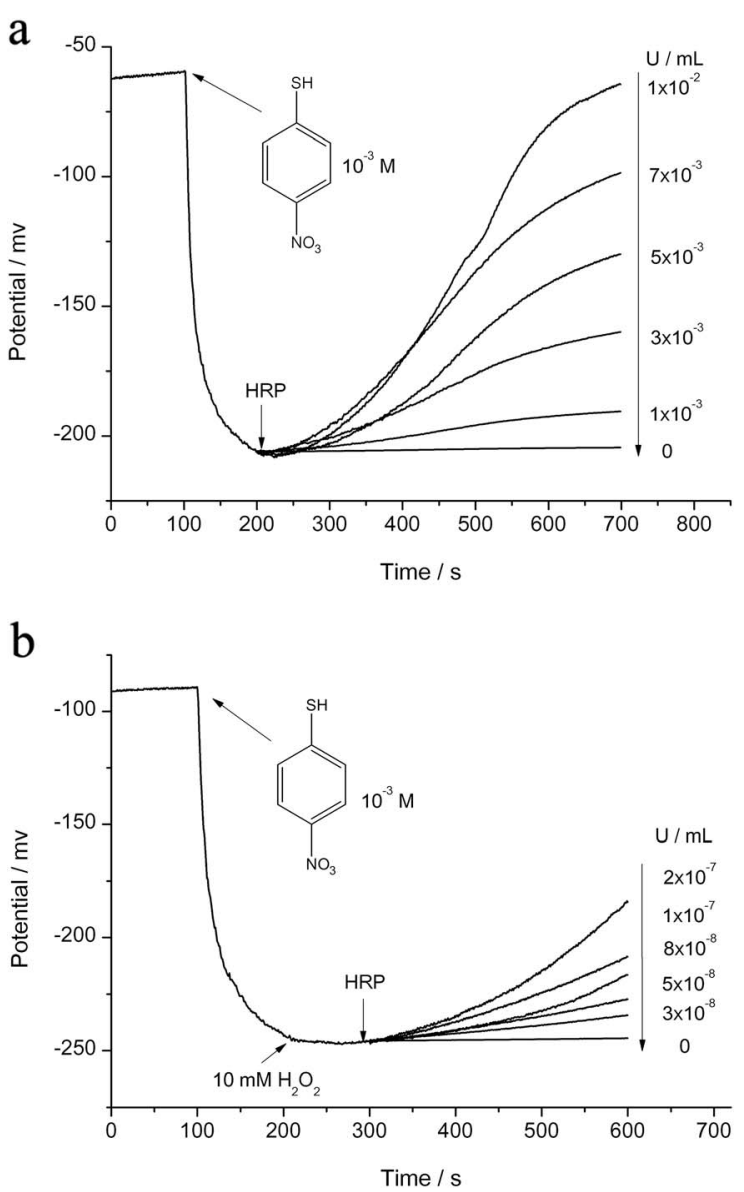

Fig. 2 Potentiometric detection of HRP by using the polymeric membrane electrode in acetate buffer $(\mathrm{pH}=4.0)$ in the absence (a) and presence (b) of $10 \mathrm{mM} \mathrm{H}_{2} \mathrm{O}_{2}$ contains $10^{-3} \mathrm{M} \mathrm{p}$-nitrothiophenol. All potential values were measured using an electrochemical workstation under zero current conditions. 
(Fig. 2). It has been reported that thiols can be oxidized to disulfides via the thiol-mediated autocatalytic generation of $\mathrm{H}_{2} \mathrm{O}_{2}$ from oxygen. ${ }^{14}$ As shown in Fig. 2(a) and Fig. S6(a) (ESI $\dagger$ ), HRP can be detected in the range of $1 \times 10^{-3}-1 \times 10^{-2} \mathrm{U} \mathrm{mL}^{-1}$ with a detection limit of $4 \times 10^{-4} \mathrm{U} \mathrm{mL}^{-1}$ in the absence of $\mathrm{H}_{2} \mathrm{O}_{2}$. Such sensitivity is comparable to traditional colorimetric method for HRP detection. It has been shown that $\mathrm{H}_{2} \mathrm{O}_{2}$ assists the oxidization of thiols and thus can improve the sensitivity for HRP detection. ${ }^{14}$ Indeed, the presence $10 \mathrm{mM} \mathrm{H}_{2} \mathrm{O}_{2}$ allows HRP to be detected in the range of $3 \times 10^{-8}-2 \times 10^{-7} \mathrm{U} \mathrm{mL}^{-1}$ with a detection limit of $2 \times 10^{-8} \mathrm{U} \mathrm{mL}^{-1}$ (Fig. 2(b) and Fig. S6(b) $(\mathrm{ESI} \dagger))$. The detection limit is 1.5 orders of magnitudes lower than that of the method based on the oxidation of $o$-phenylenediamine. ${ }^{15}$ Besides HRP detection, this versatile platform can also be used for other peroxidases and peroxidase mimics (e.g., hemin/G-quadruplexes) and their related biosensing applications.

\section{Conclusions}

In conclusion, we report here for the first time the unexpected anionic potentiometric responses to electrically neutral ArSHs of the polymeric membranes doped with lipophilic anion exchangers. The complexations between ArSHs and quaternary ammonium salts were studied in homogeneous organic solutions. The association constants between the ArSHs and $\mathrm{TDMA}^{+} \mathrm{Cl}^{-}$obtained from titration experiments correlate with the Hammett constants for the para substituents and with the response magnitudes of the ArSHs. The heterogeneous two phase extraction experiments confirm the dissociation of ArSHs in the organic phase and the ejection of $\mathrm{H}^{+} \mathrm{X}^{-}$into the aqueous phase. The response mechanism for ArSHs is similar to those of ArOHs and boronic acids. The anion exchanger doped polymeric membranes can be readily used to distinguish ArSHs from aliphatic thiols. Based on the HRP-catalyzed oxidations of ArSHs, a sensitive and facile potentiometric platform for HRP has been developed, which can be extended for applications in peroxidase/peroxidase mimetic related biosensing.

\section{Acknowledgements}

This work was financially supported by the National Natural Science Foundation of China (21475148) and the Taishan Scholar Program of Shandong Province.

\section{Notes and references}

1 (a) E. Bakker, P. Buhlmann and E. Pretsch, Chem. Rev., 1997, 97, 3083-3132; (b) J. Bobacka, A. Ivaska and A. Lewenstam, Chem. Rev., 2008, 108, 329-351; (c) P. Buhlmann and L. D. Chen, Ion-Selective Electrodes with Ionophore-Doped Sensing Membranes in Supramolecular Chemistry: From Molecules to Nanomaterials, Wiley, 2012.

2 T. Ito, H. Radecka, K. Tohda, K. Odashima and Y. Umezawa, J. Am. Chem. Soc., 1998, 120, 3049-3059.

3 X. W. Wang, D. F. Yue, E. G. Lv, L. Wu and W. Qin, Anal. Chem., 2014, 86, 1927-1931.

4 X. W. Wang, Z. F. Ding, Q. W. Ren and W. Qin, Anal. Chem., 2013, 85, 1945-1950.

5 H. D. Chen, L. Li, H. M. Guo, X. W. Wang and W. Qin, RSC Adv., 2015, 5, 13805-13808.

6 X. M. Shao, R. X. Kang, Y. L. Zhang, Z. T. Huang, F. F. Peng, J. Zhang, Y. Wang, F. C. Pan, W. J. Zhang and W. L. Zhao, Anal. Chem., 2015, 87, 399-405.

7 (a) J. C. Love, L. A. Estroff, J. K. Kriebel, R. G. Nuzzo and G. M. Whitesides, Chem. Rev., 2005, 105, 1103-1169; (b) J. Yin, Y. Kwon, D. Kim, D. Lee, G. Kim, Y. Hu, J.-H. Ryu and J. Yoon, J. Am. Chem. Soc., 2014, 136, 5351-5358; (c) H. S. Jung, X. Chen, J. S. Kim and J. Yoon, Chem. Soc. Rev., 2013, 42, 6019-6031; (d) L. A. Herzenberg, S. C. DeRosa, J. G. Dubs, M. Roederer, M. T. Anderson, S. W. Ela, S. C. Deresinski and L. A. Herzenberg, Proc. Natl. Acad. Sci. U. S. A., 1997, 94, 1967-1972.

8 J. Li, C.-F. Zhang, S.-H. Yang, W.-C. Yang and G.-F. Yang, Anal. Chem., 2014, 86, 3037-3042.

9 (a) H. Maeda, H. Matsuno, M. Ushida, K. Katayama, K. Saeki and N. Itoh, Angew. Chem., Int. Ed., 2005, 44, 2922-2925; (b) A. Taglieber, H. Höbenreich, J. D. Carballeira, R. J. G. Mondière and M. T. Reetz, Angew. Chem., Int. Ed., 2007, 46, 8597-8600.

10 P. Buhlmann, M. Hayakawa, T. Ohshiro, S. Amemiya and Y. Umezawa, Anal. Chem., 2001, 73, 3199-3205.

11 K. Odashima, T. Ito, K. Tohda and Y. Umezawa, Chem. Pharm. Bull., 1998, 46, 1248-1253.

12 C. Hansch, A. Leo and A. W. Taft, Chem. Rev., 1991, 91, 165195.

13 A. J. Fry, J. Org. Chem., 2013, 78, 5476-5481.

14 E. Golub, R. Freeman and I. Willner, Anal. Chem., 2013, 85, 12126-12133.

15 X. W. Wang and W. Qin, Chem.-Eur. J., 2013, 19, 9979-9986. 